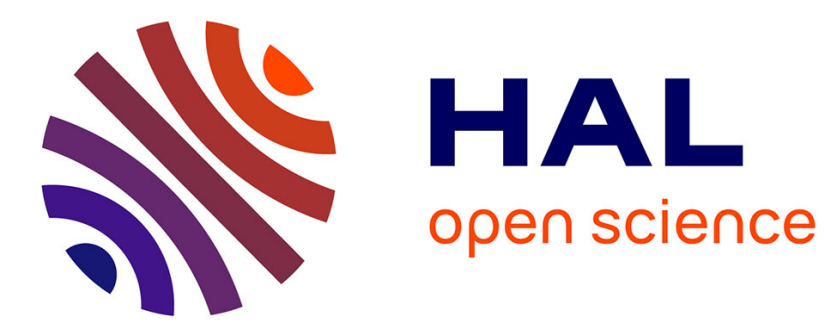

\title{
Étude de la permittivité effective de matériaux composites
}

\author{
Bruno Sareni
}

\section{To cite this version:}

Bruno Sareni. Étude de la permittivité effective de matériaux composites. Journal de Physique III, 1997, 7 (4), pp.793-801. 10.1051/jp3:1997150 . jpa-00249612

\section{HAL Id: jpa-00249612 https://hal.science/jpa-00249612}

Submitted on 1 Jan 1997

HAL is a multi-disciplinary open access archive for the deposit and dissemination of scientific research documents, whether they are published or not. The documents may come from teaching and research institutions in France or abroad, or from public or private research centers.
L'archive ouverte pluridisciplinaire HAL, est destinée au dépôt et à la diffusion de documents scientifiques de niveau recherche, publiés ou non, émanant des établissements d'enseignement et de recherche français ou étrangers, des laboratoires publics ou privés. 


\title{
Étude de la permittivité effective de matériaux composites
}

\author{
B. Sareni $\left(^{*}\right)$ \\ CEGELY $\left({ }^{* *}\right)$, École Centrale de Lyon, BP163, 69131 Ecully Cedex, France
}

(Reçu le 24 mai 1996, accepté le 18 décembre 1996)

PACS.77.20.Ch - Permittivity, dielectric function

PACS.02.70.-C - Computational techniques

\begin{abstract}
Résumé. - Dans cet article, la méthode des équations intégrales de frontières et la méthode des éléments finis sont appliquées au calcul de la permittivité effective de matériaux composites à structure périodique ou désordonnée. Les principaux paramètres physiques qui conditıonnent les propriétés macroscopiques de ces composites diélectriques sont identıfiés et étudiés. Les résultats obtenus sont comparés à ceux dérıvés de l'approche traditıonnelle du milieu effectif.
\end{abstract}

\begin{abstract}
In this paper, boundary elements and finite elements are applied to compute the effective permittıvity of perıodic or random composite materials. The main physical parameters which determıne the macroscopic properties of these dielectric composites are identified and studied. The obtained results are compared with those deduced from the traditional effective medium approach.
\end{abstract}

\section{Introduction}

L'intérêt porté aux matériaux composites s'est accru ces dernières années, en raison de leur capacité à améliorer la sensibilité et la sélectivité de dıvers dispositifs. De nouvelles applications orientées vers l'aérospatiale, l'aéronautique et les télécommunications se sont largement développées. Il s'agit en particulier des matériaux électromagnétiques absorbants, des milieux multicouches et des polymères chargés. Bien que l'étude des milieux hétérogènes ait débuté il y a plus de cent ans lorsque les scientifiques essayaient de développer le concept de champ moléculaire dans la théorie des diélectriques, la caractérisation de ces structures reste toujours délicate à cause du nombre de paramètres qu'il est nécessaire de contrôler et d'identifier. Nous présentons ici nos résultats concernant la permittivité effective de composites diélectriques à deux phases dont la structure est soit périodique, soit désordonnée. Le calcul de la permittivité est effectuée à partir d'un modèle numérique basé sur la méthode des éléments finis en $2 \mathrm{D}$ et sur la méthode des équations intégrales de frontière en $3 \mathrm{D}$.

\section{Théorie du milieu effectif}

Une grandeur effective statique telle que la permittivité, la perméabilité ou la conductivité est une quantité attribuable aux milieux hétérogènes lorsqu'à l'échelle des longueurs, ils peuvent

$\left({ }^{*}\right)$ e-mail : sareni@trotek.ec-lyon.fr

$\left({ }^{* *}\right)$ UPRESA CNRS 5005

(C) Les Éditions de Physique 1997 
être considérés comme homogènes. Cette approche n'est valable que pour des régimes quasistatiques où la longueur de corrélation $\xi$ des inhomogénéités est très inférieure à la longueur l'onde $\lambda$ du champ électrique incident $(\xi \ll \lambda / 2 \pi)[1]$.

Considérons un matériau contenant $N$ inclusions par unité de volume, chacune de permittivité $\varepsilon_{1}$ et de polarisabilité $\alpha$, dispersées dans un milieu continu de permittivité $\varepsilon_{2}$. Le déplacement électrique moyen $\bar{D}$ est directement proportionnel au champ moyen macroscopique $\bar{E}$ et le coefficient de proportionnalité définissant la permittivité effective $\varepsilon$ peut être écrit de la manière suivante :

$$
\varepsilon=\varepsilon_{2}+N \alpha \frac{\bar{E}_{1}}{\bar{E}^{2}}
$$

où le champ local $\bar{E}_{1}$ peut être calculé d'une manière générale à partir de la décomposition de Yaghjian [1]

$$
\bar{E}_{1}=\bar{E}+\frac{1}{\varepsilon_{2}} \overline{\bar{L}} \cdot N \alpha \bar{E}_{1}
$$

où $\overline{\bar{L}}$ désigne le tenseur dépolarisation des inclusions.

- Si les inclusions sont orientées arbitrairement et dispersées aléatoirement, le milieu est alors isotrope. La permittivité effective, la dépolarisation et la polarisabilité sont des constantes scalaires respectivement $\varepsilon, L$, et $\alpha$. La permittivité effective est déduite de ce qui précède :

$$
\varepsilon=\varepsilon_{2}+\frac{N \alpha \varepsilon_{2}}{\varepsilon_{2}-N \alpha L}
$$

- Si les inclusions sont dissymétriques et orientées dans une même direction, le milieu est anisotrope. La permittivité effective, la dépolarisation et la polarisabilité sont des tenseurs diagonaux respectivement $\overline{\bar{\varepsilon}}, \overline{\bar{L}}$, et $\overline{\bar{\alpha}}$. La relation précédente reste toutefois valable pour chacune de leur composante

$$
\varepsilon_{\imath}=\varepsilon_{2}+\frac{N \alpha_{\imath} \varepsilon_{2}}{\varepsilon_{2}-N \alpha_{\imath} L_{\imath}} \quad i=x, y, z
$$

Pour calculer la permittivité effective, les équations (3) et (4) impliquent la connaissance de la polarisabilité des inclusions. Celle-ci peut être déduite de l'expression du champ interne ou externe à une inhomogénéité à partir de la résolution de l'équation de Laplace $\Delta V=0$. Des expressions analytiques approchées peuvent être déterminées lorsque les inclusions ont une forme particulière (sphérique, cylindrique, ellipsoïdale, ...). Malgré tout, cette approche n'est valable que pour de faibles concentrations de la phase dispersée, celle-ci étant incapable de tenir compte de toutes les interactions entre les hétérogénéités. La méthode analytique classique est en outre limitée exclusivement à des inclusions isolées à cause des problèmes associés aux conditions d'interfaces en cas de contact ou de fusion des impuretés dans le milieu continu.

\section{Calcul numérique de la permittivité effective}

La permittivité effective peut être obtenue à partir de la résolution de l'équation de Laplace par des méthodes numériques telles les Éléments Finis (EF) ou les Équations Intégrales de Frontière (EIF). Notre choix s'est naturellement orienté vers les éléments finis en $2 \mathrm{D}$ et vers les équations intégrales de frontières en $3 \mathrm{D}$ en fonction des logiciels de calcul de champ disponibles au CEGELY (fissure, phi3D).

Considérons un composite diélectrique périodique à deux phases. En prenant en compte les propriétés de symétrie et de périodicité, la géométrie du matériau est réduite à une cellule 


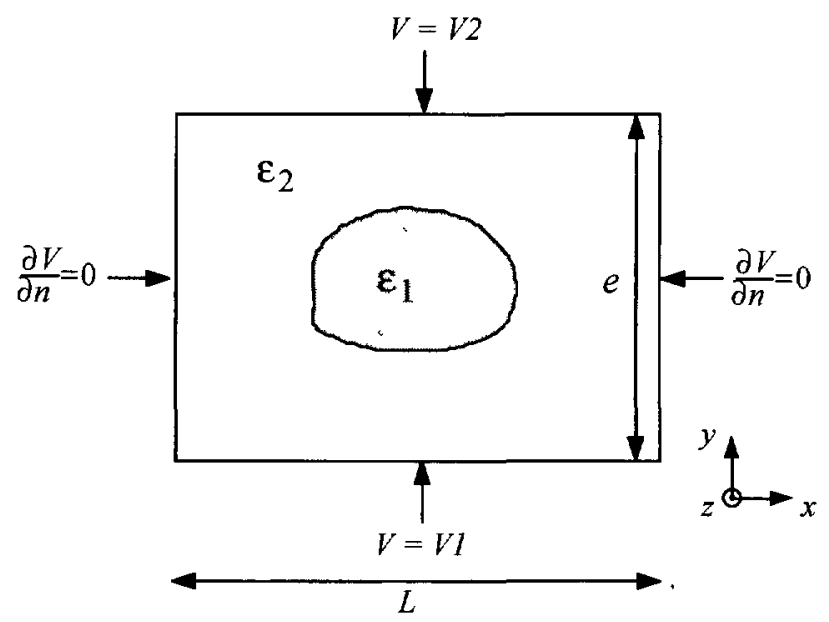

Fig. 1. - Modélisation d'un composite pérodique 2D par EF

[2D finte element model for periodic composites.]

élémentaire. En 2D, l'énergie électrostatique est calculée à partir des valeurs des dérivées du potentiel $V$ aux nœuds du maillage par $\mathrm{EF}$, sur toute la surface $S$ du composite suivant

$$
E_{\mathrm{e}}=\frac{1}{2} \iint_{S} \varepsilon(x, y)\left[\left(\frac{\partial V}{\partial x}\right)^{2}+\left(\frac{\partial V}{\partial y}\right)^{2}\right] \mathrm{d} x \mathrm{~d} y
$$

La permittivité effective $\varepsilon_{\imath}$ dans la direction correspondant au champ appliqué ( $\imath=x$ ou $\left.y\right)$ est obtenue à partir de l'énergıe électrostatique dissipée dans le condensateur plan équivalent au composite

$$
E_{\mathrm{e}}=\frac{1}{2} \varepsilon_{2} \frac{S_{\mathrm{p}}}{e}\left(V_{2}-V_{1}\right)^{2}
$$

où $e, V_{1}$, et $V_{2}$ sont définies par la figure 1. $S_{p}=L \times p$ est la surface des armatures, $p$ la profondeur du composite (en 2D, $p=1 \mathrm{~m}$ ).

Pour un composite diélectrique 3D à deux phases, la permittivité est calculée par EIF à partir de la somme des flux du déplacement électrique à travers la face de la cellule élémentaire perpendiculaire au champ appliqué :

$$
\int_{S_{1}} \varepsilon_{1} \frac{\partial V}{\partial n} \mathrm{~d} S+\int_{S_{2}} \varepsilon_{2} \frac{\partial V}{\partial n} \mathrm{~d} S=\varepsilon_{z} \frac{V_{2}-V_{1}}{e}\left(S_{1}+S_{2}\right)
$$

où $e, S_{1}, S_{2}, V_{1}$, et $V_{2}^{\top}$ sont définies par la figure $2\left(\varepsilon_{z}\right.$ étant la permittivité effective dans la direction du champ appliqué $z$ ). Ce modèle est général dans le sens où il permet de calculer la permittivité effective pour de fortes concentrations de la phase dispersée en supposant que les inhomogénéités peuvent "fusionner" entre elles, laissant de faibles espaces poreux de permittivité $\varepsilon_{2}$. Le milieu de permittivité $\varepsilon_{1}$ intercepte alors une ou plusieurs faces de la cellule élémentaire $\left(S_{1} \neq 0\right)$. Dans le cas plus classique où les inhomogénéités sont isolées dans le milıu continu, l'équation ( 7 ) se simplifie en fixant $S_{1}=0$. 


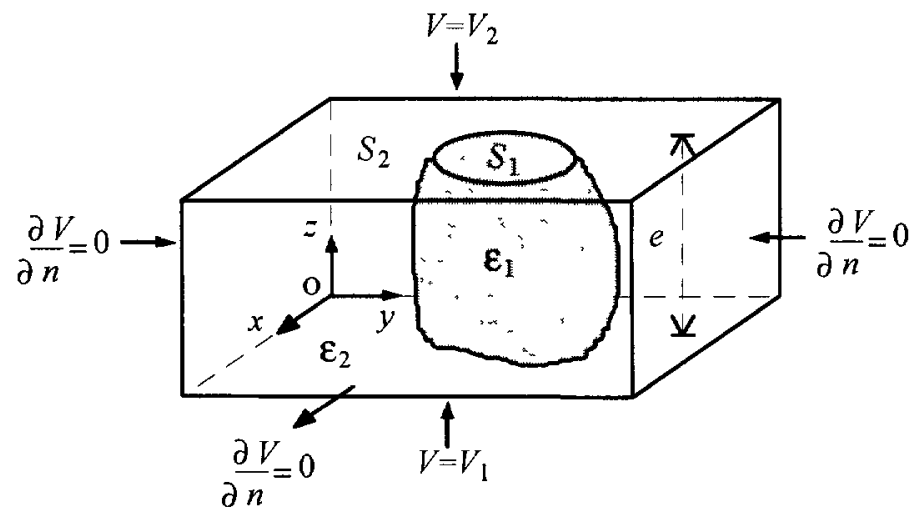

Fig. 2. - Modélisation d'un composite périodique 3D par EIF.

[3D boundary element model for periodic composite.]

\section{Permittivité effective de composites périodiques}

Considérons un composite périodique à inclusions sphériques de permittivité $\varepsilon_{1}$ régulièrement espacées à la manière d'un réseau cristallin cubique simple dans un milieu hôte de permittivité $\varepsilon_{2}$. La cellule élémentaire du composite est constituée d'une sphère de rayon $r$, centrée à l'intérieur d'un cube de côté $l$. Au-delà du seuil de percolation $(r>0,5)$ la géométrie du matériau est décrite conformément au modèle de Shen [2] en excluant de la cellule élémentaire les six calottes sphériques intérceptant le cube de côté $l$. La permittivité effective du milieu peut être évaluée pour de faibles valeurs de la fraction volumique $f$ des inclusions par la formule de Rayleigh [3]

$$
\frac{\varepsilon-\varepsilon_{2}}{\varepsilon+2 \varepsilon_{2}}=f \frac{\varepsilon_{1}-\varepsilon_{2}}{\varepsilon_{1}+2 \varepsilon_{2}}
$$

Les résultats provenant de nos simulations par EIF sont similaires à ceux obtenus récemment par une technique de développement en série de Fourier [2,4] sur l'intervalle complet de la fraction volumique $f$. Ils confirment la limitation du modèle analytique classique de Rayleigh aux faibles concentrations de la phase dispersée $(f<0,35)$ en particulier lorsque le rapport des permittivités des deux milieux est élevé (voir Fig. 3 ).

De la même manière, nous avons étudié la permittıvité effective d'un composite périodique à inclusions ellipsoïdales (sphérö̈des allongés de demi-axes $b=c=a / 4$ ), les inhomogénéités étant toutes orientées dans la direction $x$ (direction du demi grand axe $a$ ). Dans ce cas, le composite est anisotrope $\left(\varepsilon_{z}=\varepsilon_{y}\right.$ mals $\left.\varepsilon_{x} \neq \varepsilon_{z}\right)$ et la permittivité dans les 3 directions $x, y$ et $z$ est donnée par la formule de Sillars [3]

$$
\varepsilon_{\imath}=\varepsilon_{2}\left(1+\frac{\left(\varepsilon_{1}-\varepsilon_{2}\right) f}{\varepsilon_{2}+\left(\varepsilon_{1}-\varepsilon_{2}\right)(1-f) n_{\imath}}\right) ; \quad i=x, y, z .
$$

La permittivité effective du milieu, calculée par l'équation précédente et déterminée par EIF, est représentée figure 4. La différence entre les modèles analytiques et numériques est très marquée en particulier dans la direction $x$ où l'interaction entre les particules est plus importante (effet de pointe du champ électrique aux extrémités des ellipsoïdes et effet de proximité accru).

La permittivité effective de composites à inclusions cylindriques (disques de faible épaisseur et barreaux allongés) a été étudiée et comparée avec les modèles classiques de la littérature [5], ceux-ci étant toujours limités par la concentration en inclusions. 


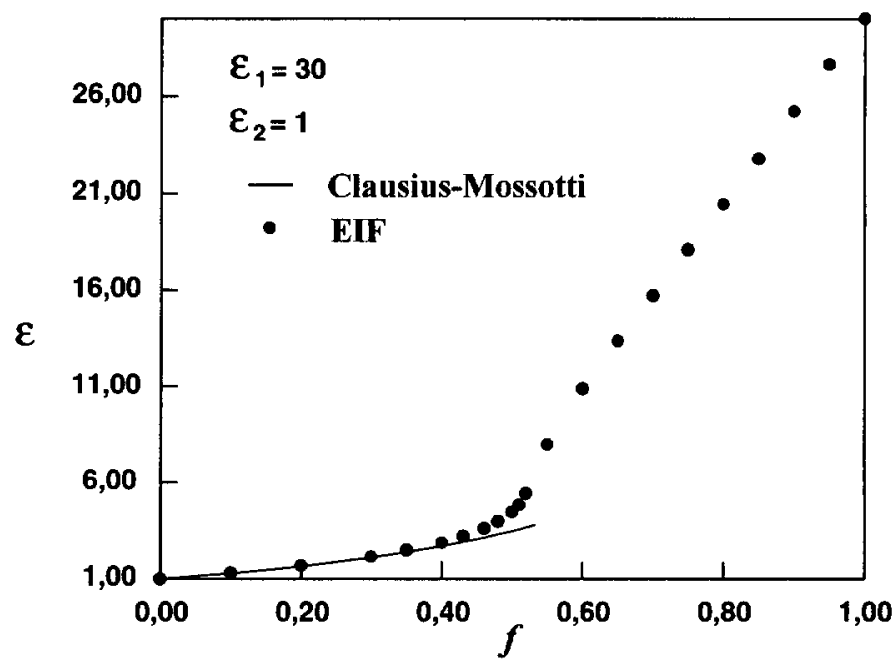

Fıg. 3. - Permittivité effective d'un composite périodique à inclusions sphériques (réseau cubique simple).

[Effective permittivity of a periodic composite with spherical inclusions (simple cubic lattice).]

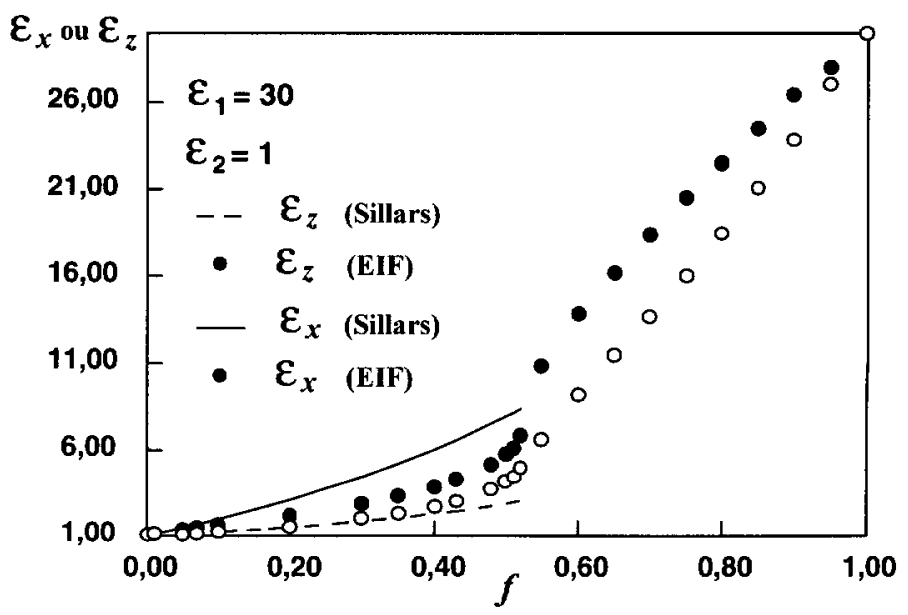

Fıg 4. - Permittivité effective d'un composıte périodıque à inclusıons ellıpsoidales de demı-axes $b=c=a / 4$.

[Effectıve permittıvity of a periodic composite with ellipsoıdal inclusions of semiaxes $b=c=a / 4$ ]

Nous montrons enfin l'influence de l'arrangement périodique des inhomogénéités sur la permittivité effective. Celle-ci peut en effet dépendre du type de réseau cristallin considéré La figure 5 illustre la permittivité de sphères conductrices parfaites placées dans un milieu hôte de permittivité $\varepsilon_{2}=1$ dans le cas de réseaux cubique simple (cs), cubique centré (cc) et cubique face centrée (cfc). 


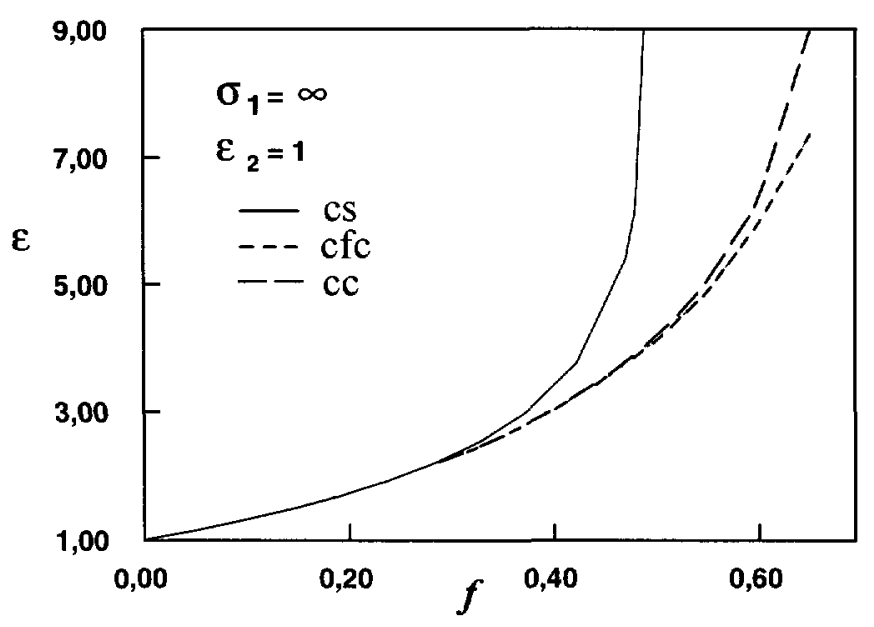

Fıg. 5. - Permittivité de réseaux cubıques de sphères conductrices parfaites.

[Effective permittivity of cubic lattices of perfectly conducting spheres.]

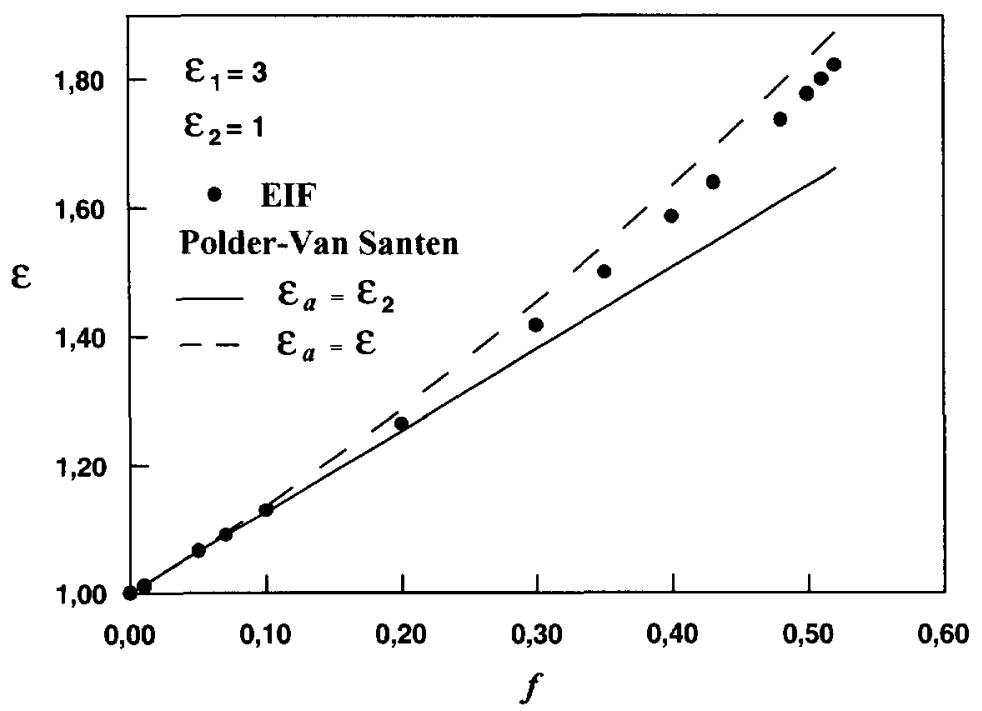

Fig. 6. - Permittivité effective d'un composite à inclusions ellipsoïdales orientées arbitrairement.

[Effective permittıvity of a random composite with ellipsoidal inclusions.]

\section{Permittivité effective de composites à structure désordonnée}

Contrairement aux milieux hétérogènes périodiques, il est très difficile d'évaluer de manière rigoureuse la constante diélectrique effective de composites désordonnés du fait du caractère aléatoire de la géométrie. Pourtant, ce type de structure correspond d'avantage à la réalité, en particulier pour des mélanges diélectriques. Les méthodes numérıques par EF et EIF utilisées jusqu'à présent sont alors peu adaptées. En effet, la description de la géométrie de milieux désordonnés nécessite la modélisation d'un grand nombre de cellules élémentaires, ce qui conduit à 


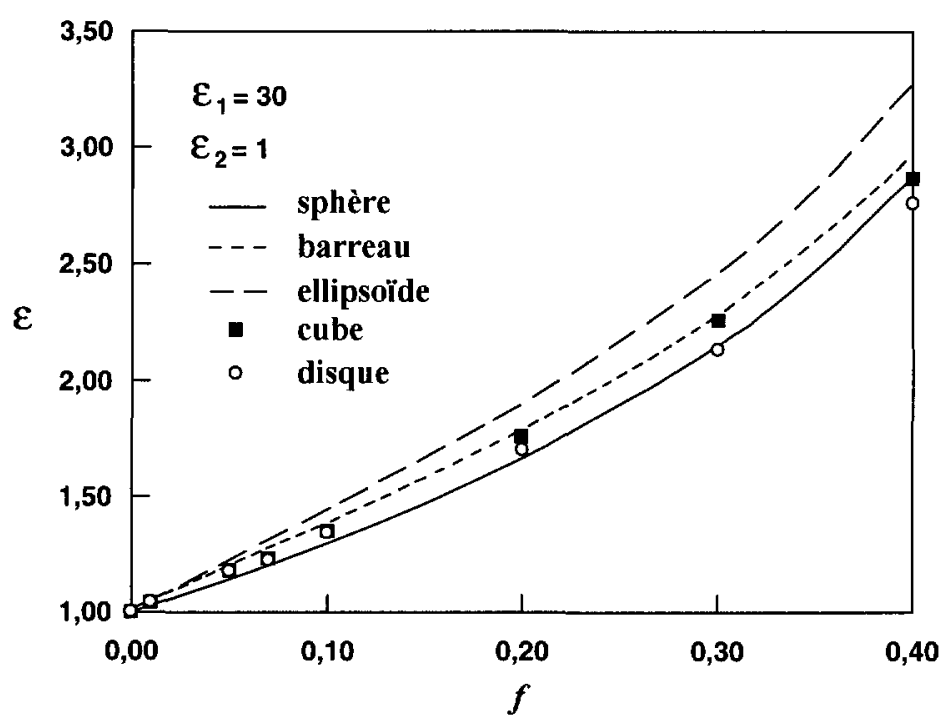

Fig 7. - Influence de la forme des inclusions sur la permittivité effectıve du milieu.

[Influence of the shape of the inclusions on the effective permittivity.]

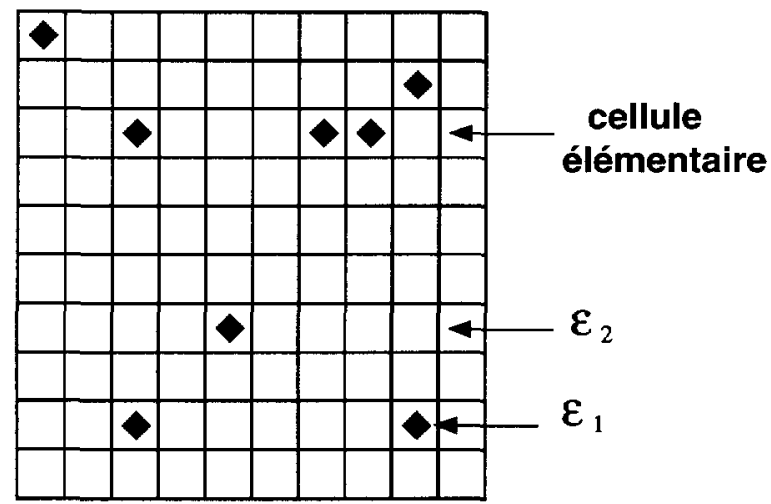

Fig 8. - Influence de la distribution des inclusions. Modèle géométrique.

[Influence of the arrangement of the inclusions. Geometric model]

une augmentation rapide du nombre d'inconnues notamment en $3 \mathrm{D}$. Pour paller à cet inconvénient, il est toujours possible de réduire la densité de maillage, mais la précision s'en trouve alors affectée. Nous tenterons ici d'évaluer qualitativement par des méthodes simples l'influence sur la permittivité effective de l'orientation, la forme et la distribution des hétérogénéités dans des mélanges diélectriques à deux constituants.

Il est possible d'approcher la permittivité effective de composites à inclusions orientées arbitrairement en partant du fait que pour ce type de structure, le milieu est nécessairement isotrope Ainsi, à partir de résultats déduits pour un composite périodique et en particulier du tenseur de permittivité $\overline{\bar{\varepsilon}}$, on calculera la permittivité scalaire $\varepsilon$ du milieu à inclusions de même nature (mais orientées arbitrairement) en prenant la moyenne des permittivités dans les 


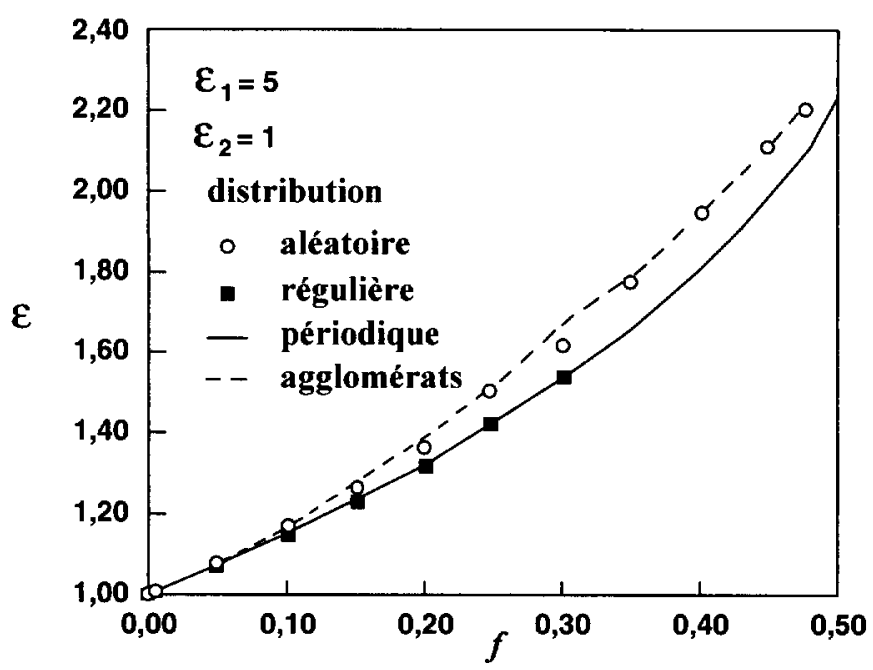

Fig. 9. - Influence de la distribution des inclusions sur la permittivité effective du milieu.

[Influence of the arrangement of the inclusions on the effective permittivity.]

trois directions $x, y$, et $z$ :

$$
\varepsilon=\frac{1}{3} \operatorname{tr} \overline{\bar{\varepsilon}}=\frac{1}{3}\left(\varepsilon_{x}+\varepsilon_{y}+\varepsilon_{z}\right) .
$$

Considérons un mélange à inclusions ellipsoïdales orientées arbitrairement : il existe de nombreuses formules analytiques décrivant la permittivité du milieu, la plus connue étant celle de Polder Van Santen $[1,3]$

$$
\varepsilon=\varepsilon_{2}+\frac{1}{3}\left(\varepsilon_{1}-\varepsilon_{2}\right) f \sum_{\imath=x, y, z} \frac{\varepsilon_{\mathrm{a}}}{\varepsilon_{\mathrm{a}}+\left(\varepsilon_{1}-\varepsilon_{\mathrm{a}}\right) n_{\imath}}
$$

où $\varepsilon_{\mathrm{a}}$ désigne la permittivité apparente du milieu continu. Sa valeur n'est pas nécessairement $\varepsilon_{2}$ mais est comprise dans l'intervalle $\varepsilon_{2} \leq \varepsilon_{\mathrm{a}} \leq \varepsilon[1,3]$.

Les résultats déduits de l'équation (10) à partir des données calculées par EIF pour un composite périodique à inclusions ellipsoidales sont présentés figure 6. L'accord entre les modèles analytique et numérique montre que l'approximation de la permittivité effective du milieu par une moyenne géométrique des composantes du tenseur de permittivité permet une bonne caractérisation des propriétés diélectriques du composite.

L'influence de la forme des inclusions sur la permittivité effective des composites est illustrée par la figure 7. L'introduction d'inhomogénéités de permittivité supérieure à celle du milieu hôte tend à augmenter la permittivité effective d'autant plus que celles-ci ont une forme allongée (ellipsoïdes, barreaux). Cette dépendance n'a pas été constatée lorsque le rapport des permittivités des deux milieux est faible (très inférıeur à 10).

Outre l'orientation et la forme des inclusions, la répartition de celles-ci dans le milieu conditionne la permittivité du composite. L'influence de la distribution des inhomogénéités est analysée en $2 \mathrm{D}$ en dupliquant plusieurs cellules élémentaires d'un composite périodique mais en dispersant les inclusions selon une concentration $C$, définie par le rapport du nombre d'inclusions sur le nombre total de cellules (voir Fig. 8).

La permittivité effective est alors déterminée par EF pour trois types de distribution des inhomogénéités : une distribution régulière quasi équivalente au composite périodique (faible 
désordre), une distribution en agglomérats où les inclusions se regroupent entre elles, et une distribution aléatoire. Les résultats obtenus montrent que le désordre a tendance à modifier les caractéristiques diélectriques du milieu, en particulier lorsque les inclusions sont regroupées. Dans ce cas, l'écart de permittivité par rapport au composite périodique est maximal, du fait d'une perturbation du champ électrique plus importante (voir Fig. 9).

\section{Conclusions}

Les éléments finis et les équations intégrales de frontières permettent de décrire avec précision la permittivité effective des matériaux composites et confirment les limitations des modèles analytiques classiques. Ces méthodes numériques peuvent être aisément adaptées à des milieux à plus de deux phases et peuvent aussi prendre en compte les pertes diélectriques pour le calcul de la permittivité effective complexe. Notre étude a dégagé les principaux paramètres qui conditionnent la permittivité effective en particulier lorsque les phases ont des caractéristiques diélectriques opposées. Enfin, par analogie physique, les résultats obtenus pour des valeurs de permittivité en électrostatique sont généralisables à des valeurs de perméabilité en magnétostatique et de conductivité en électrocinétique.

\section{Bibliographie}

[1] Sihvola A. and Kong J.A., Effective Permittivity of Dielectric Mixtures, IEEE Trans. on Geosci. and Remote Sensıng. 26 (1988) 420-429.

[2] Shen L.C., Liu C., Korringa J. and Dunn K.J., Computation of Conductivity and Dielectric Constant of Periodic Porous Media, J. Appl. Phys. 67 (1990) 7070-7081.

[3] Van Beek L.K.H., Dielectric Behaviour of Heterogeneous Systems, Progress in Dielectrics 7 (1967) 69-114.

[4] Tao R., Chen Z. and Sheng P., First-principles Fourier Approach for the Calculation of the Effective Dielectric Constant of Periodic Composites, Phys. Rev. B 3 (1989) 2417-2420.

[5] Sareni B., Krähenbühl L., Beroual A. and Brosseau C., Effective Dielectric Constant of Periodic Composite Materials, J. Appl. Phys. 80 (1996) 1688-1696. 